\title{
Coupling the symmetrical pair of temporary pulses propagating in the focusing Kerr media
}

\author{
Szymon Lasota, Jerzy Jasiński* \\ Faculty of Physics, Warsaw University of Technology, Koszykowa 75, 00-662 Warszawa,
}

Received September 14, 2009; accepted September 30, 2009; published September 30, 2009

\begin{abstract}
This work presents the analytical description of coupling two pulses propagating in phase one after the other. To do so the canonical method was applied. All pulses parameters are assumed to depend on the distance between them. Corresponding Euler-Lagrange equations are derived and solved. Explicit formulas describing oscillations of pulse parameters are obtained.
\end{abstract}

The slowly varying envelope $U(z, t)$ of temporal pulses propagating in $z$-direction in Kerr media is described by the non-linear Schrödinger equation (NLSE) [1]:

$$
\frac{\partial U}{\partial z}+\frac{i k_{2}}{2} \frac{\partial^{2} U}{\partial t^{2}}-i \varepsilon_{2}|U|^{2} U=0 \text {. }
$$

Let us multiply the above equation by $U^{*}$ and then add the complex conjugate of the received expression. Integrating the result over time we obtain $d P / d z=0$ with:

$$
P=\int_{-\infty}^{\infty} r(z, t)^{2} d t
$$

where $r=|U|$ is the amplitude of the field. The constant quantity $P$ is proportional to the pulse power. In the paper, instead of complex amplitude $U$ we shall use amplitude and phase of the field:

$$
U(z, t)=r(z, t) \cdot \text { ex pi } \cdot[\Phi(z, t)]
$$

Let us assume both amplitude and phase to be symmetric functions of time. Moreover let us write amplitude of field $r(z, t)$ as a product of pulse height $b(z)$ and shape's function $f(z, t)$ normalized by the condition $f(z, 0)=1$. Additionally we shall take the phase with parabolic temporary profile:

$$
\begin{gathered}
r(z, t)=b(z) \cdot f(z, t), \\
\Phi_{s}(z, t)=\beta(z)+\Theta(z) \cdot t^{2} .
\end{gathered}
$$

Let us assume that shape function $f(z, t)$ depends on $z$ only via pulse width $w(z)$ changing with the distance of propagation $z$. For the symmetric pair of two pulses this function is a sum of two components. Denoting by $\tau(z) w(z)$ variable distance between pulses we have:

\footnotetext{
*E-mail: jasinski@if.pw.edu.pl
}

$$
f(z, t)=f_{0}\left(\frac{t}{w(z)}-\frac{\tau(z)}{2}\right)+f_{0}\left(\frac{t}{w(z)}+\frac{\tau(z)}{2}\right) .
$$

The nonlinear Schrödinger equation for a pulse with assumed properties is not analytically solved. Instead we shall apply an approximate method based on the EulerLagrange equations. The basic role in this method is played by the Lagrange density function [2-3]:

$$
\mathcal{L}=\frac{i}{2}\left(U \frac{\partial U^{*}}{\partial z}-U^{*} \frac{\partial U}{\partial z}\right)-\frac{k_{2}}{2}\left|\frac{\partial U}{\partial t}\right|^{2}-\frac{\varepsilon_{2}|U|^{4}}{2} .
$$

By integrating Lagrange density over time from minus to plus infinity, we get the Lagrange function:

$$
L(z)=\int_{-\infty}^{\infty} \mathcal{L}(z, t) d t
$$

Taking into account assumptions (3)-(5) we obtain:

$$
\frac{L}{4 b^{2} w}=C_{1} \beta^{\prime}-\frac{C_{2} k_{2}}{2 w^{2}}-2 C_{3} \varepsilon_{2} b^{2}+C_{4} w^{2}\left(\theta^{\prime}-2 k_{2} \theta^{2}\right) .
$$

In the right-handed expression prim ' denotes differentiation over $z$ while coefficients $C_{1}, . ., C_{4}$ are overlap integrals (they are functions of relative distance $\tau(z))$ defined as follows:

$$
\begin{aligned}
& C_{1}(\tau)=\frac{1}{4} \int_{-\infty}^{\infty}\left(f_{0}(x-\tau / 2)+f_{0}(x+\tau / 2)\right)^{2} d, x \\
& C_{2}(\tau)=\frac{1}{4} \int_{-\infty}^{\infty}\left(f_{0}^{\prime}(x-\tau / 2)+f_{0}^{\prime}(x+\tau / 2)\right)^{2} d, ; \\
& C_{3}(\tau)=\frac{1}{16} \int_{-\infty}^{\infty}\left(f_{0}(x-\tau / 2)+f_{0}(x+\tau / 2)\right)^{4} d, x \\
& C_{4}(\tau)=\frac{1}{4} \int_{-\infty}^{\infty} x^{2}\left(f_{0}(x-\tau / 2)+f_{0}(x+\tau / 2)\right)^{2} d .
\end{aligned}
$$

Analogously, we are able to receive the Hamilton function, which in the case considered in the paper is:

$$
H=\frac{2 C_{2} k_{2} b^{2}}{w}+8 C_{3} \varepsilon_{2} w b^{4}+8 C_{4} k_{2} w^{3} b^{2} \theta^{2} .
$$

Each of five evolving parameters $\beta, \theta, b, w$ and $\tau$ can be treated as a generalized coordinate $q_{i}$ satisfying the EulerLagrange equation $\partial L / \partial q_{i}-\partial\left(\partial L / \partial q_{i}{ }^{\prime}\right) / \partial z=0$. The first of 
these equations gives a relation between pulse width and height:

$$
P=4 C_{1} w b^{2}
$$

This relation is identical with the expression resulting from (2) after substituting assumptions (3)-(5). To write the next equation let us denote:

$$
J=-\frac{P \varepsilon_{2}}{k_{2}} \sqrt{\frac{C_{4}}{C_{1}}} \cdot w .
$$

Therefore, quantity $J$ is connected with the pulse width, but because of the overlap integrals in definition (12), the dependence on $\tau$ of $J(\tau)$ and $w(\tau)$ is different. Applying $J(z)=J(\tau(z))$, we obtain the second of the Euler-Lagrange equation in the form:

$$
\theta=-\frac{J^{\prime}}{2 k_{2} J} .
$$

A pair of the third and fourth Euler-Lagrange equation after eliminating $w$ and $\theta$ give two expressions describing the speed of changes of phase component $\beta^{\prime}$ and the second derivative of the introduced function $J$.

$$
\begin{gathered}
\beta^{\prime}=\frac{5 C_{3} \varepsilon_{2} b^{2}}{C_{1}}+\frac{16 C_{1} C_{2} b^{4}}{P^{2}}, \\
-\frac{J^{\prime \prime}}{2 k_{2} J}=-\frac{128 C_{1}^{4} C_{2} b^{8}}{P^{4} C_{4}}-\frac{16 C_{1}^{2} C_{3} b^{6}}{P^{2} C_{4}} .
\end{gathered}
$$

The last of the Euler-Lagrange equations gives an algebraic relation between the width of pulses $w$ and their distance $\tau$. Using $J$ instead of $w$ we have:

$$
J=\frac{\frac{d}{d \tau} \frac{C_{2} C_{4}}{C_{1}^{2}}}{\frac{d}{d \tau} \sqrt{\frac{C_{4} C_{3}^{2}}{C_{1}^{5}}}} .
$$

In this way the four parameters $b, \theta, \beta^{\prime}$ and $J$ (instead of $w$ ) are functions of the fifth $\tau=\tau(\mathrm{z})$. In order to determine them as a function of propagation distance $z$ we need to solve the second equation (14). This equation is possible to integrate once, but the form of the obtained integral is easier to receive if we write law of conservation of canonical energy $H(z)=E=$ const:

$$
\frac{k_{2} J^{\prime}(z)^{2}}{2 P \varepsilon_{2}^{2}}+V(z)=E \text {. }
$$

By analogy to mechanics, the function $V(z)$ may be treated as potential. The potential also depends on $z$ by means of distance $\tau(z)$. In the explicit form it is equal:

$$
V=\frac{1}{J} \sqrt{\frac{C_{4} C_{3}^{2}}{C_{1}^{5}}}-\frac{C_{2} C_{4}}{J^{2} C_{1}^{2}} .
$$

The law of conservation of energy in the form (16) gives us the solution in the form of inverse function $z(\tau)$ :

$$
z= \pm \frac{k_{2}}{P^{2} \varepsilon_{2}^{2}} \int_{\tau(z)}^{\tau_{p}} \frac{J^{\prime}(x) d x}{\sqrt{\frac{2 k_{2} E}{P^{3} \varepsilon_{2}^{2}}+V(x)}}
$$

To find out how the result depends on $\tau$ we should analyze relations $J(\tau)$ and $V(\tau)$. For the profile $f_{0}(\tau)=\operatorname{Sech}(\tau)$ these relations are shown in Figures 1 and 2 .

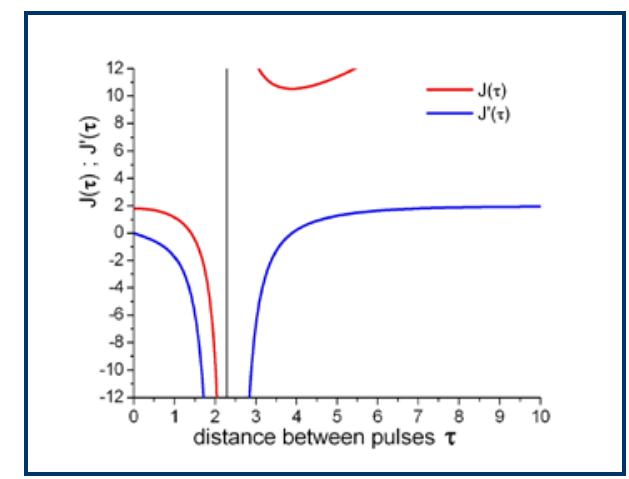

Fig.1. The variation of function $J(\tau)$ nad her derivative $J^{\prime}(\tau)$ with distance between pulses.

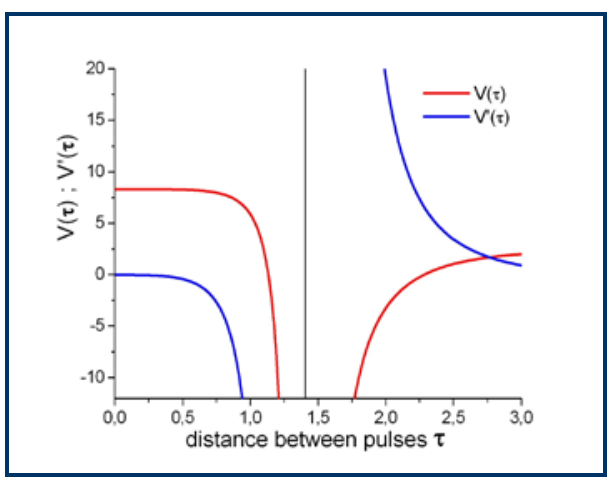

Fig.2. The variation of potential and his derivative with distance between pulses.

Having the plot $V(\tau)$ we are able to discuss qualitatively function $\tau(z)$. This function obtains physical values only when $E \leq V$. In the regime of small values, the distance $\tau(z)$ is contained between zero and a certain maximum value calculated at the intersection of the horizontal line $E=$ const and the curve $V(\tau)$. Reaching its maximum value (satisfying the equation $V\left(\tau_{\max }\right)=E$ ), the distance begins to decrease, so $\tau(z)$ oscillates. We can approximate analytical relation describing such oscillations if we expand the functions in the integrand (18) into series of small $x$. Doing so with accuracy up to $x^{4}$-order terms, we derive: 


$$
Z=-\frac{\pi k_{2}}{\sqrt{3 V_{4}} \cdot P^{2} \varepsilon_{2}^{2}} \int_{\tau}^{\tau_{p}} \frac{2 x\left(J_{2}+2 J_{4} x^{2}\right)}{\sqrt{\tau_{p}^{2}-x^{4}}} .
$$

In the above integral $\tau_{p}$ is point of return $\left(\tau_{p}=\tau_{\max }\right)$ while $J_{2}, J_{4}$ and $V_{4}$ are the corresponding coefficients of expansion of $J(x)$ and $V(x)$ into Taylor's series. For $f_{0}(\tau)=\operatorname{Sech}(\tau)$ these coefficients equals $J_{2}=0.2700$, $J_{4}=0.0748$ and $V_{4}=0.0054$.

The form (19) give us an approximate solution in the analytic form:

$$
z=-\frac{\pi k_{2}}{\sqrt{3 V_{4}} \cdot P^{2} \varepsilon_{2}^{2}}\left(J_{2} \arccos \left(\tau^{2} / \tau_{p}^{2}\right)-2 J_{4} \sqrt{\tau_{p}^{4}-\tau^{4}}\right) .
$$

In Fig. 3 we show the plot of the obtained approximate solution (20) for a few first periods. Although distance oscillations look quite similar to harmonic ones, the function $z(\tau)$ could not be inversed and its shape differs from harmonic oscillations $\tau=\tau_{\mathrm{p}} \cdot \cos (\omega z)$.

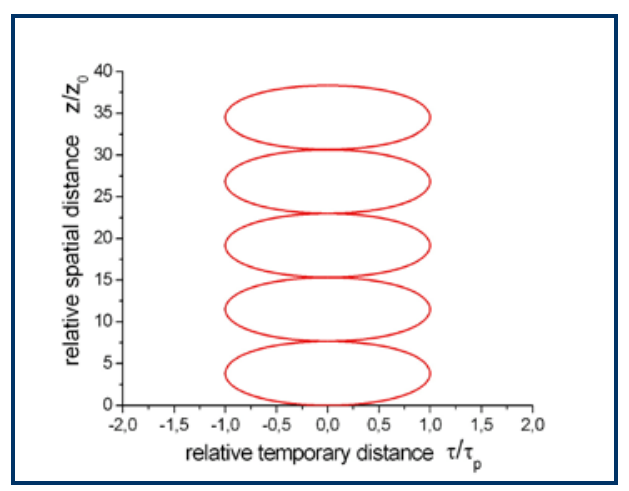

Fig.3. The variation of the relative temporary distance $\tau / \tau_{p}$ as the function of relative spatial distance $z / z_{0}$ for $\tau_{p}=1$ The distance unit $z_{0}$ equals: $z_{0}=-2 \pi k_{2} J_{4} / \sqrt{ }\left(3 V_{4}\right) P^{2} \varepsilon_{2}^{2}$.

The above solution enables us to determine the explicit formula for the period of oscillations - a quarter of this period is the distance between position $z$ corresponding to $\tau=\tau_{p}$ and the position corresponding to $\tau=0$ :

$$
\Lambda=-\frac{\pi k_{2}}{\sqrt{3 V_{4}} P^{2} \varepsilon_{2}^{2}}\left(2 \pi J_{2}+8 J_{4} \tau_{p}^{2}\right) .
$$

To find out the applicability regime of the applied method let us compare the obtained results and numeric solution of nonlinear Schrödinger equation (1) (Fig. 4). As a measure of accuracy of solutions (20) let us take the difference between the oscillation period given analytically and obtained numerically. Assume material parameters $k_{2}$ and $\varepsilon_{2}$ that characterize fused silica. For $P=122 \mathrm{kV}^{2} \cdot \mathrm{ps} / \mathrm{mm}^{2}$, initial width $w(0)=50$ fs and initial distance $\tau_{p} w(0)=62.5$ fs we have $\Lambda / 2=84.9 \mathrm{~m}$ from (21), while numeric results give $\Lambda / 2=85.2 \mathrm{~m}$ (to obtain distance oscillations better visible, Fig. 4 was plotted for

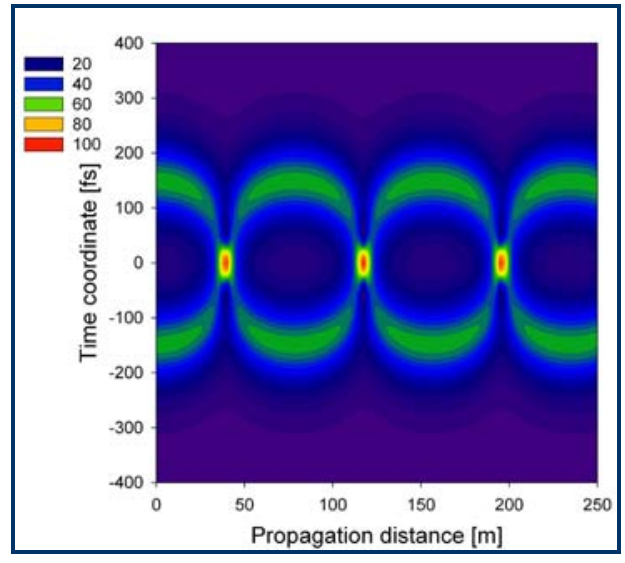

Fig.4. Numeric pulse oscillations.

much larger initial distance $\left.\tau_{p} w(0)=300 \mathrm{fs}\right)$. Therefore the analytical solution (20) approximates evolution of pulse parameters quite well, although such a good coincidence works only for small initial distances between both components, what is the key assumption to apply the Taylor expansion (19). The initial height of a single pulse $b(0)$ in the limit $\tau_{p} \rightarrow 0$ should tend to half of the height of a soliton of width $w(0)$, so the sum of two pulses is quite close to a single soliton with oscillating height and width. But even not very small value of $\tau_{p}$ (1.25 in the considered example) gives accuracy of analytical description better than $1 \%$. Unfortunately, for a bit greater initial distance the difference between solutions rapidly increases being about $30 \%$ for $\tau_{p}=1.5$. The reason of such behavior lies in the potential function - it possesses the vertical asymptote appearing for $\tau_{c r}=1.404$, as we can see in Fig. 2, so expansion like (19) breaks down.

The canonical method enable an analytical description of the evolution of a symmetric pair of temporal pulses. Applying an adequate trial function and five pulse parameters we are able to write and solve all EulerLagrange equations. As a result, we obtain a quadrature expressing the evolution of distance between pulses. Applying a small distance approximation we were able to obtain an explicit analytic formula for evolving pulse distance. The obtained analytical solution agrees quite well with numerics, although for larger initial distances the difference between an analytic and numeric solution is much higher.

\section{References}

[1] G. P. Agraval, Nonlinear Fiber Optics, (San Diego, Academic Press, 2001)

[2] G.P. Agrawal, Lightwave technology:telecommunications systems, pp. 137-142, Wiley Interscience, Hoboken, 2005

[3] D. Anderson, Phys. Rev. A, 27, 3135, (1983)

[4] H. Goldstein, C. Poole, J. Safko, Classical Mechanics, (Addison Wesley, San Francisco, 2000) 